\title{
Large cell neuroendocrine carcinoma of the lungs: case report and literature review
}

\author{
Ying Han, Lingrong Pang, Jia Huang, Jun Chen \\ Department of Chemoradiotherapy, the Affiliated People's Hospital of Ningbo University, Ningbo, China \\ Correspondence to: Ying Han. Department of Chemoradiotherapy, the Affiliated People's Hospital of Ningbo University, No. 251 Baizhang Road, \\ Ningbo, China. Email: Dr.han@vip.163.com.
}

\begin{abstract}
Pulmonary large cell neuroendocrine carcinoma (LCNEC) is a rare but destructive tumor type, accounting for approximately $1 \%$ of all lung cancers, associated with poor prognosis. LCNEC is challenging diagnosing using biopsy specimens. While current LCNEC therapies include surgery, radiotherapy, and chemotherapy, it has not yet proved its greatest treatment strategies. Immunotherapy is rapidly emerging as a possibility for lung cancer treatment. However, there are scant reports in the literature regarding LCNEC immunotherapy. Therefore, the author here reports a case of LCNEC by immunotherapy, and retrospective reviews the present research status and progress of LENCE and corresponding clinical treatment progress. This case will supply valuable information for the treatment options for LCNEC. A 64-year-old male smoker was treated for one month for blood in his sputum. Chest radiography and computed tomography revealed a 3-cm solitary tumor in the left upper lung. We treated the patient with thoracoscopic radical surgery for upper left lung cancer. Postoperative pathology shows pulmonary LCNEC. We performed postoperative chemotherapy with a double-drug regimen holding platinum. Then, bevacizumab, paclitaxel, and the PDL1 checkpoint inhibitor nivolumab were applied, but the patient progressed rapidly. Immunotherapy is an ineffective treatment possibility for these patients, even if PD-L1 expression is positive. A possible contributing factor is the timing of immunotherapy too late.
\end{abstract}

Keywords: Large cell neuroendocrine carcinoma (LCNEC); chemotherapy; target therapy; treatment options; immunotherapy

Submitted Jul 23, 2020. Accepted for publication Sep 14, 2020.

doi: 10.21037/apm-20-1667

View this article at: http://dx.doi.org/10.21037/apm-20-1667

\section{Introduction}

Pulmonary LCNEC was first described by Travis et al. (1), is a rare but highly aggressive tumor type, accounting for approximately $1 \%$ of all lung cancers. Patients with LCNEC are predominantly male (2), older, and heavy smokers. The median age is $\approx 60$ years; this disease is uncommon in non-smoking females. LCNEC is poorly symptomatic; cough, hemoptysis, and post-obstructive pneumonia are infrequent. Sometimes, patients present with asymptomatic nodules, chest pain, nonspecific flu-like symptoms, dyspnea, night sweats, or carcinoid syndrome. LCNEC is categorized as a high-grade neuroendocrine tumor, in the same category as small cell lung cancer (SCLC). In the 1970s, pulmonary neuroendocrine tumors were classified into three histologically defined categories: typical carcinoids (TC); atypical carcinoids (AC), usually defined as carcinoids; and the more undifferentiated entity represented by SCLC. Then, in 1999 and 2004, the World Health Organization recognized LCNEC as a variant of large cell carcinoma (LCC), which is a type of nonsmall cell lung cancer (NSCLC) and one of the four major types of lung neuroendocrine tumors; LCNEC is the only type of NSCLC that requires immunohistochemical (IHC) staining for at least one positive neuroendocrine marker such as chromogranin A, synaptophysin or CD56 


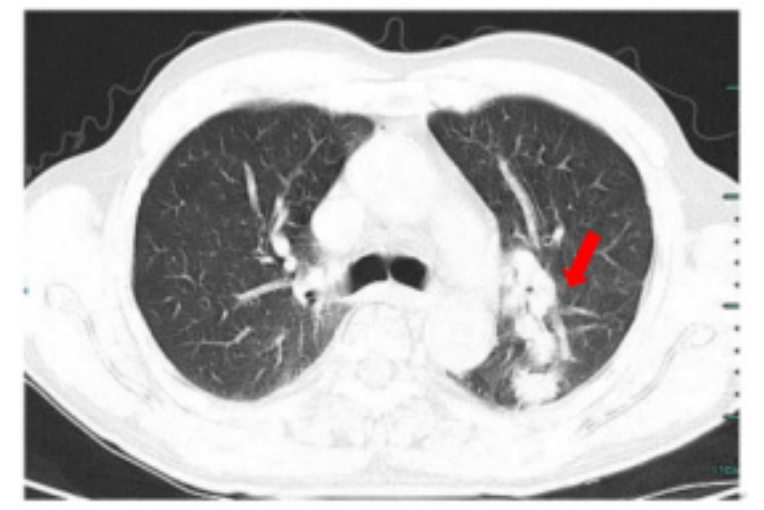

Figure 1 Chest radiography and computed tomography revealed a 3 -cm solitary tumor in the left upper lung, the lesion is identified with red arrow.

to confirm definite diagnosis (3). In most cases, LCNEC develops peripherally and is rare in the central part of the lung, causing concomitant atelectasis; metastasis often occurs in the liver, bone, brain, adrenal gland, lung, pleura and extra-thoracic lymph nodes (4). Here, we report a case of Pulmonary LCNEC. We present the following case in accordance with the CARE reporting checklist (available at http://dx.doi.org/10.21037/apm-20-1667).

\section{Case presentation}

A 64-year-old male smoker was treated for one month for blood in his sputum; he had a clear history of asbestos or silica dust exposure. He had a substantial smoking history of approximately ten cigarettes per day for 30 years. There was no history and family history. Chest radiography and computed tomography $(\mathrm{CT})$ revealed a 3 -cm solitary tumor in the left upper lung (Figure 1). Malignancy was considered clinically. Contrast-enhanced brain magnetic resonance imaging revealed no metastatic foci in the brain. We observed no bone metastasis or other distant metastases. One week after hospitalization, the patient was treated with thoracoscopic radical surgery for upper left lung cancer. Postoperative pathology shows pulmonary LCNEC, the mass is $3 \mathrm{~cm} \times 3 \mathrm{~cm} \times 2.5 \mathrm{~cm}$, group 11 lymph node see 1 metastasis. TNM is T2N1M0, stage IIB. The tumor cells show moderately abundant cytoplasm, pleomorphic and vesicular nuclei, and mitosis (up to 20 mitoses $/ 2 \mathrm{~mm}^{2}$ ). Immunohistochemical analysis showed that the tumor cells were diffusely and strongly positive for Ki-67, EMA, and neuroendocrine markers (synaptophysin, and CD56) (Figure 2) and negative for thyroid transcription factor-1, chromogranin, Napsin A, CK5/6, and CD20. We performed postoperative chemotherapy with a doubledrug regimen holding platinum. The patient received one course of postoperative adjuvant chemotherapy (etoposide, $100 \mathrm{mg} / \mathrm{m}^{2} /$ day, day $1-3$; carboplatin AUC 5-6, day 1). The patient developed hoarseness after chemotherapy and found no abnormalities during laryngoscopy. CT's examination of the chest revealed multiple enlarged lymph nodes near the left pulmonary hilum and aortic arch, metastasis was considered, and we observed left pleural effusion. Fluorodeoxyglucose positron emission tomography (FDGPET) showed: metastatic pleural tumor with left pleural effusion; left residual lung nodule shadow; multiple lymph node metastases in the mediastinum and hilum of the left lung; right adrenal metastasis, without excluding left adrenal metastasis; and systemic bone metastasis. The enlarged lymph nodes in the right iliac fossa and the left paracolic sulcus are suspected to be metastatic. Genetic testing of the surgical specimens suggested PD-L1 staining of $10 \%$. Lowlevel microsatellite instability was noted, and the tumor mutation load was 36.29. Epidermal growth factor receptor (EGFR), ALK, KRAS (-), TP53 pathogenic mutations were not observed. Subsequently, two cycles of a chemotherapy regimen holding paclitaxel $300 \mathrm{mg} \mathrm{d} 1$, cisplatin 40 $\mathrm{mg} \mathrm{d} 1-3$, and bevacizumab $500 \mathrm{mg} \mathrm{d} 1$ anti-vascular chemotherapy was administered. During chemotherapy, the patient experienced lip numbness. Contrast-enhanced brain magnetic resonance imaging revealed left parietal lobe metastases in the brain. Three months after surgery, we performed radiotherapy for metastatic brain tumors (45 Gy; 15 fractions). A reexamination of the images suggested the rapid progression of the metastatic tumors in the lungs and spine. Four months after the operation, therapy with the PD-L1 checkpoint inhibitor nivolumab (200 mg d1 q21) was started. Despite immunotherapy, the tumors in the lungs and bone and the other distant metastases progressed rapidly. The patient chose to stop treatment five months after surgery. Immunotherapy is an ineffective treatment possibility for these patients, even if $\mathrm{PD}-\mathrm{L} 1$ expression is positive.

All procedures performed in studies involving human participants were in accordance with the ethical standards of the institutional and/or national research committee(s) and 

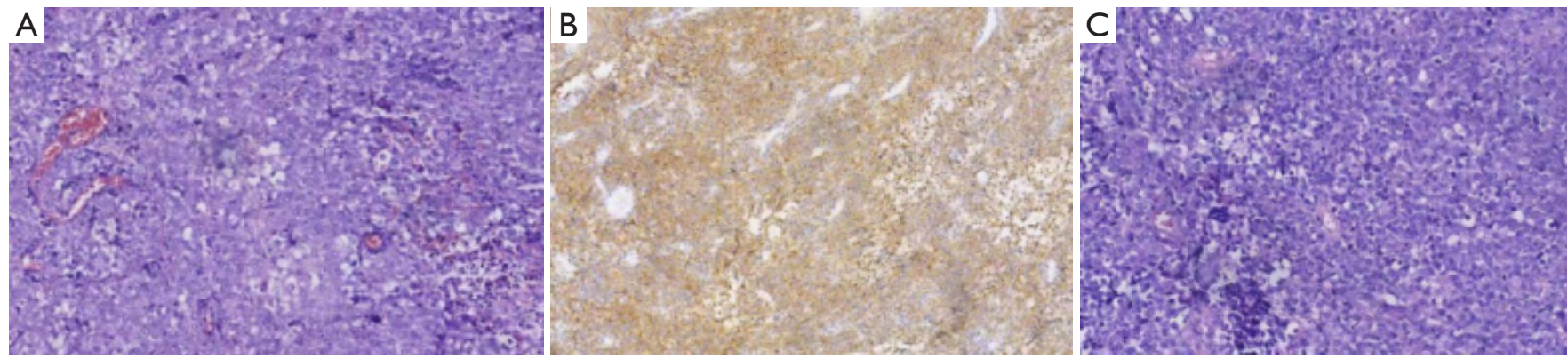

Figure 2 (A,B,C) 200× magnification. (A) The tumor cells appear large and pleomorphic with abundant cytoplasm, vesicular nuclei and large nucleoli with hematoxylin and eosin (HE) staining; (B) immunohistochemical analysis showed diffuse positive syn staining in the cytoplasm; (C) necrosis can be observed in the mitotic divisions under hematoxylin and eosin (HE) staining, up to $102 / \mathrm{mm}^{2}$.

with the Helsinki Declaration (as revised in 2013). Written informed consent was obtained from the patient.

\section{Discussion}

Our patient progressed rapidly after treatment, motivating us to review the advancements in the treatment of LCNEC. Series of surgically resected stage I-III lung cancer have shown that LCNEC and SCLC have similar prognoses. Patients with potentially resectable stage I small cell neuroendocrine carcinoma or LCNEC have 5-year survival rates ranging from $27 \%$ to $59 \%$, there is difference in the stage IV (5). Therefore, we review the progress of the treatment of advanced lung large cell neuroendocrine carcinoma (LCNEC). In the current European Society for Medical Oncology guidelines for NSCLC, no specific treatment for LCNEC is described (6). In the American Society of Clinical Oncology (ASCO) guidelines, either platinum/etoposide chemotherapy or the same regimen for NSCLC is recommended for LCNEC, example as cisplatin combined with pemetrexed (7). The optimal adjuvant and advanced chemotherapy regimen is still an existing controversy (8). Forty-nine patients with stage IV LCNEC were identified, and 37 patients had available data regarding treatment for first-line metastatic disease: $70 \%(n=26)$ received platinum/etoposide, and 30\% ( $n=11)$ received other regimens (9). Two-phase II trials, both with pathological reviews, have been reported. A European trial (10) reported progression-free survival (PFS) of 5.0 months (95\% CI: 4.0-7.9 months) and response rate (ORR) of $34 \%$ in 29 patients treated with platinum/etoposide chemotherapy. In a Japanese trial (11), a median OS of 12.6 (95\% CI: 9.3-16.0) months, PFS of 5.8 (95\% CI: 3.8-7.8) months, and ORR of $47 \%$ were reported after treatment with platinum/irinotecan $(n=30)$. Considering these results, the response of LCNEC to platinum-based chemotherapy may be comparable to SCLC and better than NSCLC.

However, in a prospective, multicenter phase II trial, chemotherapy-naive patients with stage IV LCNEC received $5 \mathrm{mg}$ everolimus daily combined with paclitaxel $175 \mathrm{mg} / \mathrm{m}^{2}$ and carboplatin AUC 5 every three weeks for a maximum of four cycles followed by maintenance everolimus $5 \mathrm{mg}$ daily until progression. The ORR was $45 \%$, the disease control rate was $74 \%$, the median PFS was 4.4 months, and the median OS was 9.9 months. The PFS rate at three months (primary endpoint) was $76 \%$, according to a Kaplan-Meier analysis (12). In another study, they categorized first-line chemotherapy into three groups, (I) group I, which comprised gemcitabine, docetaxel, paclitaxel, or vinorelbine; (II) group II, with pemetrexed treatment only; and (III) group III, which comprised etoposide chemotherapy. The median (95\% CI) OS after the group I chemotherapy was 8.5 months, which is significantly longer than that for patients treated with group II chemotherapy, who had a median survival of 5.9 months, and for patients treated with group III chemotherapy, who had a median survival of 6.7 months. In patients with LCNEC, the group I chemotherapy resulted in a longer OS than group II and group III chemotherapy (13). Therefore, new, more extensive prospective trials should be encouraged to confirm gemcitabine, docetaxel, paclitaxel, or vinorelbine or etoposide chemotherapy approaches for both diagnosed and suspected pulmonary LCNEC.

No defined biomarker of response to chemotherapy has been found. Patients with LCNEC tumors that carry a wildtype RB1 gene or express the RB1 protein respond better 
to platinum plus gemcitabine or taxanes (NSCLC-GEM/ TAX) treatment than to platinum plus etoposide (SCLCPE) chemotherapy (14). The few limitations of these studies, including their retrospective design, encourage the replication of these results in a prospective randomized clinical trial that stratifies LCNEC from genomic subtypes.

In the current research, the mutational landscape revealed that TP53, RB1, NF1, SMARCA4, EGFR, ALK, KEAP1, and NOTCH1 were the most frequently mutated genes in the cfDNA of this LCNEC patient (15-17). However, the frequency of EGFR mutations in LCNEC patients is low; in one study, genetic alterations were detected in $12(15 \%)$ tumors, with an EGFR mutation rate of $1 \%$ (18). The first report of LCNEC carrying an EGFR activating mutation and of gefitinib activity in LCNEC patients was published in July 2010; a 66-year-old woman who had never smoked was diagnosed with metastatic LCNEC. An activating EGFR mutation (exon 19, p. L747_A755>AT) was detected in the extracted DNA, and a correlation between exon 19 deletion and LCNEC response to gefitinib was reported (19). Another case harboring an EGFR mutation showed a response to an EGFR-tyrosine kinase inhibitor (EGFRTKI). Specifically, a neuroendocrine carcinoma (LCNEC) harboring an EGFR gene mutation responded for eight months to the EGFR-TKI icotinib (20). Zheng found that LCNEC responded to ALK inhibitor (crizotinib) treatment for ten months (21).

PD-L1 expression was found in 10.4-40\% of LCNEC cases. The high frequency of PD-L1 expression could support using the anti-programmed cell death 1 antibody in the treatment of LCNEC (22). A paper reported that second-line systemic therapy with nivolumab $(3 \mathrm{mg} / \mathrm{kg}$ every two weeks) was started for a tumor pathologically staged according to the TNM classification (eighth edition) as ypT0 ypN0 (R0). Nivolumab was not continued after surgery, and no other adjuvant treatment was planned. The patient was still in complete remission and free of treatment eight months after surgical resection (23). This case revealed a complete response to nivolumab after palliative thoracic radiotherapy in this rare subset of lung cancer that has previously been characterized by a poor prognosis. However, in our case, we did not observe the same efficacy. Immunotherapy is an ineffective treatment possibility for these patients, even if PD-L1 expression is positive. A possible contributing factor is the timing of immunotherapy too late, which is also this case's limitation. The efficacy of immune checkpoint inhibitors in LCNEC has not yet been thoroughly evaluated, and more research is still required to discover a breakthrough in terms of survival.

\section{Acknowledgments}

We thank Guangzhou Yujia Biotechnology Co., Ltd for helpful conversations.

Funding: None.

\section{Footnote}

Reporting Checklist: The authors have completed the CARE reporting checklist. Available at http://dx.doi.org/10.21037/ apm-20-1667

Conflicts of Interest: All authors have completed the ICMJE uniform disclosure form (available at http://dx.doi. org/10.21037/apm-20-1667). The authors have no conflicts of interest to declare.

Ethical Statement: The authors are accountable for all aspects of the work in ensuring that questions related to the accuracy or integrity of any part of the work are appropriately investigated and resolved. All procedures performed in studies involving human participants were in accordance with the ethical standards of the institutional and/or national research committee(s) and with the Helsinki Declaration (as revised in 2013). Written informed consent was obtained from the patient.

Open Access Statement: This is an Open Access article distributed in accordance with the Creative Commons Attribution-NonCommercial-NoDerivs 4.0 International License (CC BY-NC-ND 4.0), which permits the noncommercial replication and distribution of the article with the strict proviso that no changes or edits are made and the original work is properly cited (including links to both the formal publication through the relevant DOI and the license). See: https://creativecommons.org/licenses/by-nc-nd/4.0/.

\section{References}

1. Travis WD, Linnoila RI, Tsokos MG, et al. Neuroendocrine tumors of the lung with proposed criteria for large-cell neuroendocrine carcinoma. An ultrastructural, immunohistochemical, and flow cytometric study of 35 cases. Am J Surg Pathol 1991;15:529-53.

2. Ichiki $\mathrm{Y}$, Matsumiya $\mathrm{H}$, Mori $\mathrm{M}$, et al. Predictive factors of postoperative survival among patients with pulmonary 
neuroendocrine tumor. J Thorac Dis 2018;10:6912-20.

3. Travis WD, Brambilla E, Nicholson AG, et al. The 2015 World Health Organization Classification of Lung Tumors: Impact of Genetic, Clinical and Radiologic Advances Since the 2004 Classification. J Thorac Oncol 2015;10:1243-60.

4. Rindi G, Klersy C, Inzani F, et al. Grading the neuroendocrine tumors of the lung: an evidence-based proposal. Endocr Relat Cancer 2014;21:1-16.

5. Arbour KC, Riely GJ. Systemic Therapy for Locally Advanced and Metastatic Non-Small Cell Lung Cancer: A Review. Jama 2019;322:764-74.

6. Reck M, Popat S, Reinmuth N, et al. Metastatic nonsmall-cell lung cancer (NSCLC): ESMO Clinical Practice Guidelines for diagnosis, treatment and follow-up. Ann Oncol 2014;25 Suppl 3:iii27-39.

7. Tang H, Wang H, Xi S, et al. Perioperative chemotherapy with pemetrexed and cisplatin for pulmonary large-cell neuroendocrine carcinoma: a case report and literature review. Onco Targets Ther 2018;11:2557-63.

8. Zhang JT, Li Y, Yan LX, et al. Disparity in clinical outcomes between pure and combined pulmonary large-cell neuroendocrine carcinoma: A multi-center retrospective study. Lung Cancer 2020;139:118-23.

9. Naidoo J, Santos-Zabala ML, Iyriboz T, et al. Large Cell Neuroendocrine Carcinoma of the Lung: ClinicoPathologic Features, Treatment, and Outcomes. Clin Lung Cancer 2016;17:e121-9.

10. Le Treut J, Sault MC, Lena H, et al. Multicentre phase II study of cisplatin-etoposide chemotherapy for advanced large-cell neuroendocrine lung carcinoma: the GFPC 0302 study. Ann Oncol 2013;24:1548-52.

11. Niho S, Kenmotsu H, Sekine I, et al. Combination chemotherapy with irinotecan and cisplatin for large-cell neuroendocrine carcinoma of the lung: a multicenter phase II study. J Thorac Oncol 2013;8:980-4.

12. Christopoulos P, Engel-Riedel W, Grohé C, et al. Everolimus with paclitaxel and carboplatin as firstline treatment for metastatic large-cell neuroendocrine lung carcinoma: a multicenter phase II trial. Ann Oncol 2017;28:1898-902.

13. Derks JL, van Suylen RJ, Thunnissen E, et al. Chemotherapy for pulmonary large cell neuroendocrine carcinomas: does the regimen matter? Eur Respir J 2017;49:1601838.

14. Derks JL, Leblay N, Thunnissen E, et al. Molecular Subtypes of Pulmonary Large-cell Neuroendocrine Carcinoma Predict Chemotherapy Treatment Outcome.
Clin Cancer Res 2018;24:33-42.

15. Zhuo M, Guan Y, Yang X, et al. The Prognostic and Therapeutic Role of Genomic Subtyping by Sequencing Tumor or Cell-Free DNA in Pulmonary LargeCell Neuroendocrine Carcinoma. Clin Cancer Res 2020;26:892-901.

16. Simbolo M, Barbi S, Fassan M, et al. Gene Expression Profiling of Lung Atypical Carcinoids and Large Cell Neuroendocrine Carcinomas Identifies Three Transcriptomic Subtypes with Specific Genomic Alterations. J Thorac Oncol 2019;14:1651-61.

17. Zhou Z, Zhu L, Niu X, et al. Comparison of genomic landscapes of large cell neuroendocrine carcinoma, small cell lung carcinoma, and large cell carcinoma. Thorac Cancer 2019;10:839-47.

18. Miyoshi T, Umemura S, Matsumura Y, et al. Genomic Profiling of Large-Cell Neuroendocrine Carcinoma of the Lung. Clin Cancer Res 2017;23:757-65.

19. De Pas TM, Giovannini M, Manzotti M, et al. Largecell neuroendocrine carcinoma of the lung harboring EGFR mutation and responding to gefitinib. J Clin Oncol 2011;29:e819-22.

20. Wang Y, Shen YH, Ma S, et al. A marked response to icotinib in a patient with large cell neuroendocrine carcinoma harboring an EGFR mutation: A case report. Oncol Lett 2015;10:1575-8.

21. Zheng Q, Zheng M, Jin Y, et al. ALK-rearrangement neuroendocrine carcinoma of the lung: a comprehensive study of a rare case series and review of literature. Onco Targets Ther 2018;11:4991-8.

22. Kim HS, Lee JH, Nam SJ, et al. Association of PDL1 Expression with Tumor-Infiltrating Immune Cells and Mutation Burden in High-Grade Neuroendocrine Carcinoma of the Lung. J Thorac Oncol 2018;13:636-48.

23. Mauclet C, Duplaquet F, Pirard L, et al. Complete tumor response of a locally advanced lung large-cell neuroendocrine carcinoma after palliative thoracic radiotherapy and immunotherapy with nivolumab. Lung Cancer 2019;128:53-6.

(English Language Editor: J. Chapnick)

Cite this article as: Han Y, Pang L, Huang J, Chen J. Large cell neuroendocrine carcinoma of the lungs: case report and literature review. Ann Palliat Med 2020;9(5):3705-3709. doi: 10.21037/apm-20-1667 